\title{
Relación entre las necesidades de cuidado y funcionamiento familiar en mujeres afectadas por violencia en un asentamiento humano del distrito de Puente Piedra
}

\author{
Aurora Marlene Giraldo-Giraldo ${ }^{1, a}$, Julia Raquel Meléndez-De la Cruz ${ }^{1, b}$
}

\section{RESUMEN}

Objetivo: determinar la relación entre las necesidades de cuidado y el funcionamiento familiar de las mujeres afectadas por violencia intrafamiliar. Material y métodos: investigación de tipo correlacional y de corte transversal, que se realizó con 74 mujeres afectadas por violencia intrafamiliar según criterios de selección. Se utilizó un cuestionario que indagó sobre necesidades de cuidado empleando la valoración según 6 de las 14 necesidades de Virginia Henderson y el funcionamiento familiar según APGAR. Resultados: el 37,9\% tenía entre 35 a 44 años; el 73\% eran convivientes; el $67,6 \%$ eran ama de casa; el 29,7\% con secundaria incompleta. El 100\% de mujeres presenta necesidades de cuidado de seguridad y la necesidad de comunicarse. Con un funcionamiento familiar del 51,4\% con disfunción familiar severa. El coeficiente de correlación Rho de Spearman $(\mathrm{R}=-0.240)$ muestra una correlación estadísticamente significativa ( $\mathrm{p}=0.039)$, la cual resultó negativa, lo que significa que niveles bajos de necesidades de cuidado se corresponden con niveles altos de funcionamiento familiar y viceversa. Conclusiones: Existe una relación significativa entre las necesidades de cuidado y el funcionamiento familiar donde las características sociodemográficas las hace vulnerables, con necesidades de seguridad y comunicación expresando emociones y temores, con disfunción familiar severa. Palabras clave: Violencia familiar, Necesidades básicas, Funcionamiento familiar (Fuente DesCS Bireme).

\section{THE RELATIONSHIP BETWEEN CARE NEEDS AND FAMILY FUNCTIONING OF WOMEN AFFECTED BY VIOLENCE}

\begin{abstract}
Objective: To determine the relationship between care needs and family functioning of women affected by domestic violence. Material and methods: The study correlational and cross-sectional was conducted with 74 women affected by domestic violence as selection criteria. It was a questionnaire that inquired about using care needs assessment as 6 of the 14 Virginia Henderson needs and family functioning as APGAR. Results: $37.9 \%$ were between $35-44$ years; $73 \%$ were cohabiting; $67.6 \%$ were housewives; $29.7 \%$ of elementary education. $100 \%$ of women develop care needs security and the need to communicate. With a familiar operation of $51.4 \%$ with severe family dysfunction. The correlation coefficient Spearman $\mathrm{Rho}(\mathrm{R}=0.240)$ showed a statistically significant correlation $(\mathrm{p}=0.039)$, which was negative, which means that low levels of care needs correspond to high levels of family functioning and vice versa. Conclusions: There is a significant relationship between care needs and family functioning where the sociodemographic characteristics makes them vulnerable to security and communication needs of expressing emotions and fears, with severe family dysfunction.
\end{abstract}

Keywords: Family violence, Basic Needs, family functioning.

Facultad de Enfermería, Universidad Peruana Cayetano Heredia. Lima - Perú.

Magister en Gestión del Cuidado en Enfermería. ${ }^{\mathrm{b}}$ Magister en Salud Pública. 


\section{INTRODUCCIÓN}

La violencia familiar es definida por la OMS como "aquella acción que ocurre en el marco de las relaciones familiares, ejercida individual o grupalmente, de manera, deliberada o no, mediante el uso de la fuerza con el propósito de infringir daño y/o imponer una forma de pensar, sentir y actuar al interior de la familia; afectando física, psicológica y sexualmente, hasta restar o destruir su potencialidad de realización y de vida" (1).

La violencia contra la mujer, se entiende como todo acto de violencia basada en su condición de género que tenga o pueda tener como resultado un daño o sufrimiento físico, sexual o psicológico para la mujer, así como las amenazas de tales actos, la coacción o la privación arbitraria de la libertad; tanto si se producen en la vida pública como en la vida privada (2).

Se considera que la violencia en las familias peruanas es una realidad, un hecho que raramente nos es ajeno, forma parte de nuestras experiencias cotidianas y la mayoría de las veces una presencia invisible según se evidencia en el documento "Brechas de Género: insumos para la adopción de medidas a favor de las mujeres" 2010; en el que concluyen que es un atentado contra los derechos humanos, afecta el desarrollo de la mujer, vulnera derechos como la vida, integridad personal, física y psicológica, derecho a la salud, a la seguridad personal, causando secuelas en los demás integrantes de la familia (3).

En el Perú la Encuesta demográfica y de salud familiar (ENDES) realizada el año 2013; indica que el 71,5\% de las mujeres alguna vez unidas sufrieron algún tipo de violencia por parte del esposo o compañero y entre las formas de violencia que se dan en el seno familiar destaca la violencia psicológica y/o verbal el 67,5\%; la violencia física el $35,7 \%$; que se presenta cuando se causa daño en la integridad física de la víctima y el $69,8 \%$ de las mujeres alguna vez unidas fueron maltratadas físicamente, solo el $14,0 \%$ acudieron al establecimiento de salud (4-5).

En este contexto la violencia infligida por la pareja en el entorno familiar se define como una práctica de dominación y exclusión social que surge como prolongación de los modelos patriarcales al interior de la familia, que en muchas ocasiones tiende a autorregularse convirtiéndose en el mecanismo de canalización de la angustia del agresor y en gran medida del núcleo familiar, así, la violencia se concentra principalmente en las instituciones sociales y se prolonga a través de los sistemas de identificación comunitaria hacia la familia por medio del lenguaje, el encuentro social, los imaginarios colectivos, las actitudes y los ataques internos y externos que sufra el sistema familiar. Los hombres que golpean se caracterizan en general por necesidad de control, conflictos de dependencia, miedo a la intimidad y antecedentes de familias violentas (6).

Las mujeres afectadas por violencia estuvieron expuestas en su niñez a circunstancias de violencia psicológica, las mujeres que poseen una autoestima baja muestran una mayor tolerancia a la violencia, el uso de la violencia por parte de la pareja es independiente de la participación económica de la mujer en el hogar; sin embargo, constituye un factor social que agrava la situación de violencia en las mujeres dedicadas a las labores domésticas, lo que las condiciona a estar expuestas a la violencia psicológica al pedir apoyo a sus parejas, fomenta un clima intimidatorio y limitan el espacio social de la víctima, influyendo en el desencadenamiento de la violencia (7).

Se tomará el concepto de necesidad del modelo de Virginia Henderson que define necesidad como "Todo aquello que es esencial al ser humano para mantener su vida o asegurar su bienestar", requisito que condiciona la supervivencia absoluta de una persona. Considerando que las mujeres víctimas de violencia intrafamiliar infligida por la pareja tienen necesidades no cubiertas sobre todo en el plano afectivo. Se tomará el concepto de necesidad como un modo, una forma, una práctica, un proceso, con actitudes $\mathrm{y}$ estrategias formales e informales, visibles, sentidas, percibidas o no, porque las necesidades van encaminadas a recuperar lo perdido, ocasionado por la situación de violencia que configura a través de la salud de los seres humanos en su proceso de vivir, como respuesta a las necesidades básicas según la teoría de Virginia Henderson (8).

En ese sentido el cuidado de enfermería a las víctimas de la violencia debe ser diseñado para promover la seguridad, aceptación, el respeto y la satisfacción de sus necesidades individuales. El cuidado requiere enfermeras que empleen herramientas básicas para práctica profesional, que involucran la observación, la atención emocional, el toque terapéutico, el cuerpo, el sentido común, el liderazgo, el carácter humanitaria, la solidaridad, la sensibilidad, la técnica, la relación educativa y las dimensiones psicosociales y psico espiritual, estableciendo una relación de cuidado. En una relación de cuidado es necesario un proceso interactivo entre el cuidador y la persona que está siendo atendido, para que esto ocurra se compara intencionalidad necesaria, disponibilidad, la apertura, la confianza y la promoción de la aceptación del ser humano en los aspectos biológico, psicológico, social y espiritual (9). 
Se eligió el Modelo de Virginia Henderson porque es el que guía la práctica de la mayoría de las enfermeras en el área sanitaria. Este modelo aporta un análisis en el que la persona es única y compleja con 14 necesidades básicas, las cuales están determinadas por aspectos biológicos, psicológicos, socioculturales y espirituales. El modo de satisfacerlas depende de: la edad, el sexo, etapa de desarrollo, situación de vida y salud, cultura, entorno y de las diferentes experiencias vividas. La función de la enfermera es ayudar en la satisfacción de estas necesidades cuando no tiene fuerza, voluntad o conocimientos necesarios para llevarlo a cabo por sí sola. De forma que le ayude a recobrar la independencia lo más rápidamente posible (10).

Para fines del estudio se abordarán 6 de las 14 necesidades, que pertenecen a las necesidades psicológico-sociales como la necesidad de seguridad, de comunicación, de creencias y valores, de trabajar y realizarse, de actividades recreativas y aprendizaje; que son indispensables para mantener la armonía e integridad de la persona. Cada necesidad está influenciada por los componentes biológicos, psicológicos, socioculturales y espirituales. Las necesidades interactúan entre ellas, cada persona las satisface y manifiesta de una manera: Cuidados básicos (11).

El funcionamiento familiar, es aquel que posibilita a la familia cumplir exitosamente con los objetivos y funciones que le están histórica y socialmente asignados, entre los cuales podemos citar los siguientes: la satisfacción de las necesidades afectivo-emocionales, la transmisión de valores éticos y culturales, la promoción y facilitación del proceso de socialización de sus miembros, el establecimiento y mantenimiento de un equilibrio que sirva para enfrentar las tensiones que se producen, el establecimiento de patrones para las relaciones interpersonales (la educación para la convivencia social), la creación de condiciones propicias para el desarrollo de la identidad personal y la adquisición de la identidad sexual (12).

El APGAR familiar es un instrumento diseñado en 1978 por el Dr. Gabriel Smilkstein (Universidad de Washington) y modificada por la Dra. Liliana Arias, quien propuso la aplicación de este test como un instrumento para los equipos de atención primaria, en su aproximación al análisis de la función familiar. Este test se basa en la premisa de que los miembros de la familia perciben el funcionamiento de la familia y pueden manifestar el grado de satisfacción con el cumplimiento de sus parámetros básicos, evaluando cinco funciones básicas de la familia, consideradas las más importantes como son: $(13,14)$.
La adaptación, evalúa la forma en que los miembros de la familia, utilizan los recursos intra y extra familiares en los momentos de grandes necesidades y periodos de crisis, para resolver sus problemas y adaptarse a las nuevas situaciones. La participación, mide la satisfacción de cada miembro de la familia, en relación con el grado de comunicación existente, entre ellos en asuntos de interés común y en la búsqueda de estrategias y soluciones para sus problemas. La ganancia, hace referencia a la forma en que los miembros de la familia encuentran satisfacción en la medida en que su grupo familiar acepte y apoye las acciones que emprendan para impulsar y fortalecer su crecimiento personal. El afecto, evalúa la satisfacción de cada miembro de la familia en relación a la respuesta ante expresiones de amor, afecto, pena o rabia, dentro del grupo familiar. Los recursos, evalúa la satisfacción de cada miembro de la familia, en relación a la forma en que cada uno de ellos se compromete a dedicar tiempo, espacio y dinero a los demás.

Considerando que constituye una problemática latente, continuamos con barreras que limitan la intervención oportuna, sobre todo en la atención primaria, asimismo, son escasos los estudios previos. Aplicando una valoración según Virginia Henderson se generará evidencia para poder intervenir en el marco de la salud integral, por lo que el estudio tiene por objetivo determinar la relación entre las necesidades de cuidado y el funcionamiento familiar de las mujeres afectadas por violencia intrafamiliar.

\section{MATERIAL Y MÉTODO}

Investigación correlacional y de corte transversal. Se realizó en el 2014 en el Asentamiento Humano Ampliación "Jesús de Oropeza Chonta" Zapallal - distrito de Puente Piedra. La técnica de recolección de datos fue la entrevista y como instrumento la encuesta demográfica de salud familiar ENDES 2010 - sección 10 que se aplicó al total de la población en el Asentamiento Humano Ampliación "Jesús Oropeza Chonta" de esta manera se determinó la población de estudio encontrando 74 mujeres afectadas por violencia intrafamiliar.

Los criterios de inclusión fueron: mujeres entre 18 a 60 años que acepten voluntariamente participar en el estudio, que estén viviendo una situación de violencia intrafamiliar y son víctimas de algún tipo de violencia física, psicológica o sexual por parte de la pareja; cónyuge, ex cónyuge, conviviente, ex conviviente. Como criterio de exclusión, mujeres que presenten alguna dificultad para responder al instrumento de la investigación.

Se cuidó en todo momento la seguridad de las mujeres que participaron en el estudio, garantizando su participación 
voluntaria, no fueron identificadas por otras personas ajenas a la investigación, se hizo uso de seudónimos, se evitó cualquier daño a su integridad por parte del agresor, siendo el lugar de la aplicación de la entrevista: el centro de salud y el comedor popular de la zona.

El instrumento fue sometido a pruebas que demostraron su validez y confiabilidad, para la variable necesidades de cuidado de Virginia Henderson el instrumento para efectos del estudio priorizó 6 de las 14 necesidades de cuidado y para la variable Funcionamiento familiar se aplicó el APGAR, instrumento validado que es útil para evidenciar la forma en que una persona percibe el funcionamiento de su familia en un momento determinado como parte de las características propias de la familia.

El proyecto fue aprobado por el Comité Institucional de Ética de la Universidad Peruana Cayetano Heredia.

Concluida la recolección de la información, los datos fueron codificados e ingresados a una base de datos creada en el programa estadístico SPSS versión 19.0 y se obtuvieron los resultados, para determinar la relación entre las variables de estudio se aplicó el coeficiente de correlación Rho de Spearman, para la identificación de las características sociodemográficas, necesidades de cuidado y para la variable funcionamiento familiar se empleó estadística descriptiva univarida, también se empleó el intervalo de confianza al $95 \%$, y se presentaron los hallazgos en tablas de frecuencia.

\section{RESULTADOS}

Tabla 1. Características sociodemográficas de las mujeres afectadas por violencia intrafamiliar en un asentamiento humano del distrito de Puente Piedra.

\begin{tabular}{|l|l|l|l|}
\hline $\begin{array}{c}\text { CARACTERISTICAS } \\
\text { SOCIODEMOGRAFICAS }\end{array}$ & & $\mathbf{N}^{\mathbf{0}}$ & $\mathbf{\%}$ \\
\hline \multirow{5}{*}{ GRUPO ETÁREO } & 18 a 24 & 9 & 12,2 \\
\cline { 2 - 4 } & 25 a 29 & 9 & 12,2 \\
\cline { 2 - 4 } & 30 a 34 & 10 & 13,5 \\
\cline { 2 - 4 } & 35 a 39 & 12 & 17,6 \\
\cline { 2 - 4 } & 40 a 44 & 15 & 20,3 \\
\cline { 2 - 4 } & 45 a 49 & 6 & 8,1 \\
\cline { 2 - 4 } & 50 a 60 & 13 & 16,2 \\
\hline \multirow{5}{*}{ ESTADO CIVIL } & Casada & 9 & 12,2 \\
\cline { 2 - 4 } & Conviviente & 54 & 73,0 \\
\cline { 2 - 4 } & Separada & 9 & 12,2 \\
\cline { 2 - 4 } & Viuda & 1 & 1,4 \\
\cline { 2 - 4 } & Soltera & 1 & 1,4 \\
\hline
\end{tabular}

\begin{tabular}{|l|l|l|l|}
\hline \multirow{5}{*}{ OCUPACION } & Ama de casa & 50 & 67,6 \\
\cline { 2 - 4 } & $\begin{array}{l}\text { Trabajo } \\
\text { independiente }\end{array}$ & 11 & 14,9 \\
\cline { 2 - 4 } & $\begin{array}{l}\text { Trabajo } \\
\text { dependiente }\end{array}$ & 5 & 6,8 \\
\cline { 2 - 4 } & $\begin{array}{l}\text { Trabajo } \\
\text { eventual }\end{array}$ & 7 & 9,5 \\
\cline { 2 - 4 } & Estudiante & 1 & 1,4 \\
\hline \multirow{5}{*}{$\begin{array}{l}\text { GRADO DE } \\
\text { INSTRUCCIÓN }\end{array}$} & $\begin{array}{l}\text { Primaria } \\
\text { incompleta }\end{array}$ & 17 & 23,0 \\
\cline { 2 - 4 } & $\begin{array}{l}\text { Primaria } \\
\text { completa }\end{array}$ & 14 & 16,9 \\
\cline { 2 - 4 } & $\begin{array}{l}\text { Secundaria } \\
\text { incompleta }\end{array}$ & 22 & 29,7 \\
\cline { 2 - 4 } & $\begin{array}{l}\text { Secundaria } \\
\text { completa }\end{array}$ & 16 & 21,6 \\
\cline { 2 - 4 } & $\begin{array}{l}\text { Superior } \\
\text { incompleta }\end{array}$ & 3 & 4,1 \\
\cline { 2 - 4 } & $\begin{array}{l}\text { Superior } \\
\text { completa }\end{array}$ & 2 & 2,7 \\
\hline
\end{tabular}

Respecto a las características socio demográficas que el $20,3 \%$ y $17,6 \%$ de mujeres se encuentran entre las edades de 40 a 44 años y 35 a 39 años respectivamente, un $73,0 \%$ de las entrevistados son convivientes; el 29.7\% tiene secundaria incompleta; así mismo, el 67,6\% se desempeñan como amas de casa.

Tabla 2. Necesidades de cuidado en mujeres afectadas por violencia intrafamiliar en el asentamiento humano del distrito de Puente Piedra.

\begin{tabular}{|c|c|c|c|c|c|}
\hline \multirow[t]{2}{*}{$\begin{array}{l}\text { NECESIDADES DE } \\
\text { CUIDADO }\end{array}$} & \multicolumn{2}{|c|}{$\begin{array}{l}\text { Manifestación } \\
\text { de satisfacción }\end{array}$} & \multicolumn{2}{|c|}{$\begin{array}{c}\text { Manifestación } \\
\text { de } \\
\text { insatisfacción }\end{array}$} & \multirow[t]{2}{*}{ TOTAL } \\
\hline & $\mathrm{N}^{\mathrm{o}}$ & $\%$ & $\mathrm{~N}^{\mathrm{o}}$ & $\%$ & \\
\hline $\begin{array}{l}\text { Necesidad de seguridad/ } \\
\text { evitar peligros. }\end{array}$ & 0 & 0 & 74 & 100.0 & 74 \\
\hline $\begin{array}{l}\text { Necesidad de comunicarse } \\
\text { (expresar emociones, } \\
\text { temores). }\end{array}$ & 0 & 0 & 74 & 100.0 & 74 \\
\hline $\begin{array}{l}\text { Necesidad de vivir según } \\
\text { sus creencias y valores. }\end{array}$ & 2 & 2.7 & 72 & 97.3 & 74 \\
\hline $\begin{array}{l}\text { Necesidad de trabajar y } \\
\text { realizarse. }\end{array}$ & 7 & 9.5 & 67 & 90.5 & 74 \\
\hline $\begin{array}{l}\text { Necesidad de participar en } \\
\text { actividades recreativas. }\end{array}$ & 28 & 37.8 & 46 & 62.2 & 74 \\
\hline Necesidad de aprendizaje. & 32 & 43.2 & 42 & 56.8 & 74 \\
\hline
\end{tabular}

En relación a las necesidades de cuidado se puede observar que las manifestaciones de insatisfacción que prevalecieron fueron la necesidad de seguridad/ 
evitar peligros y la necesidad de comunicarse (expresar emociones, necesidades, temores u opiniones).

Tabla 3. Funcionamiento familiar en mujeres afectadas por violencia intrafamiliar en el asentamiento humano del distrito de Puente Piedra.

\begin{tabular}{|l|l|l|l|l|}
\hline \multirow{2}{*}{$\begin{array}{l}\text { FUNCIONAMIENTO } \\
\text { FAMILIAR }\end{array}$} & \multirow{2}{*}{$\mathbf{N}^{\mathbf{0}}$} & $\mathbf{\%}$ & $\begin{array}{l}\text { Lim. } \\
\text { Inferior }\end{array}$ & $\begin{array}{c}\text { Lim. } \\
\text { Superior }\end{array}$ \\
\hline $\begin{array}{l}\text { Buen funcionamiento } \\
\text { familiar }\end{array}$ & 1 & 1,4 & 0,0 & 4,1 \\
\hline Disfunción familiar leve & 8 & 10,8 & 4,1 & 18,9 \\
\hline $\begin{array}{l}\text { Disfunción familiar } \\
\text { moderada }\end{array}$ & 27 & 36,5 & 25,7 & 48,6 \\
\hline Disfunción familiar severa & 38 & 51,4 & 39,2 & 62,2 \\
\hline Total & 74 & $100 \%$ & & \\
\hline
\end{tabular}

De acuerdo al funcionamiento familiar (Apgar) se observa que del total de entrevistadas un considerable $51.4 \%$ presentó disfunción familiar severa.

Grafico 1. Relación entre las necesidades de cuidado $\mathrm{y}$ funcionamiento familiar en mujeres afectadas por violencia intrafamiliar en $\mathrm{n}$ asentamiento humano del distrito de Puente Piedra.

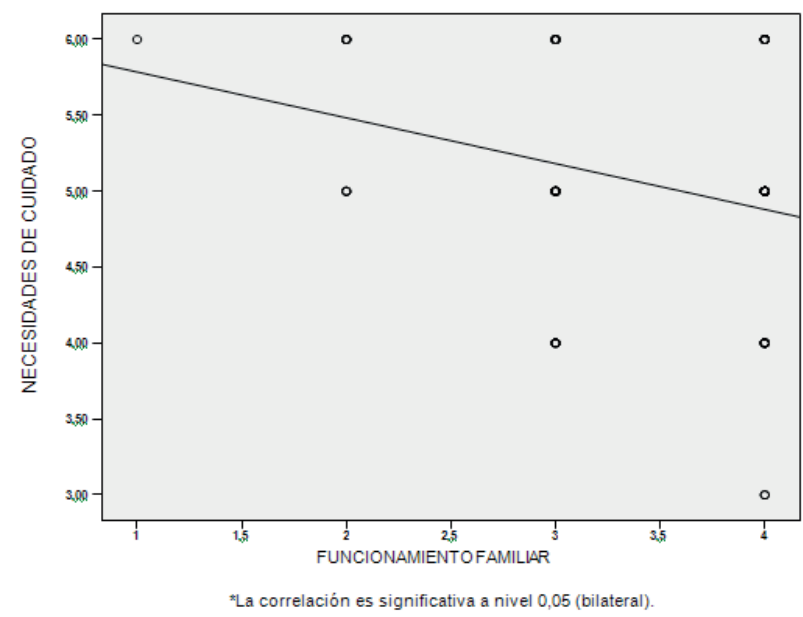

En el grafico 1, el coeficiente de correlación Rho de Spearman $(\mathrm{Rho}=-0.240)$ muestra una correlación estadísticamente significativa $(\mathrm{p}=0.039)$ entre las necesidades de cuidado y el funcionamiento familiar.

\section{DISCUSIÓN}

El estudio se realizó en una zona geográfica ubicada en el distrito de Puente Piedra al cono norte de Lima, que concentra a varios asentamientos humanos y representa uno de los más grandes cinturones de pobreza, donde las mujeres son el grupo más vulnerable. Cabe resaltar que en el Centro de Emergencia Mujer en Puente Piedra se registraron 182 casos de mujeres afectadas por violencia familiar con edades entre 18 a 59 años, en el período de enero a junio 2014.

Presentan características socio demográficas que las vulneran. ENDES (2013) coincide con el hallazgo respecto a que la violencia familiar por parte de la pareja es más frecuente en mujeres adultas de 25 a 29 y de 45 a 49 años de edad (4).

Acerca del grado de escolaridad predominaron las mujeres que tienen secundaria incompleta, semejante resultado de Bardales quien señala que a mayor nivel educativo menor porcentaje de violencia contra la mujer (15). Estos hallazgos refuerzan lo encontrado en el estudio y es necesario abordar el nivel educativo de las mujeres para garantizar un menor riesgo a ser vulnerada y que le permita buscar mejores redes de apoyo.

Con respecto al estado civil, los resultados pueden ser explicados debido a que en la actualidad la convivencia tiene un mayor grado de aceptación como opción de vida en pareja y por encima del compromiso en matrimonio, ello plantea al Estado el deber de intervenir para mejorar en términos de legalidad y asegurar mecanismos de protección para las mujeres y sus hijos frente a la eventualidad de separación o disolución del vínculo (16).

La mayoría de entrevistadas tienen como ocupación ama de casa, estuvieron más expuestas a la violencia porque, dependen económicamente de la pareja, constituye un factor social que agrava la situación de violencia en las mujeres dedicadas a las labores domésticas. En ese sentido estos resultados respaldan la generación de desigualdad de oportunidades con dependencia económica de la mujer dedicada a labores domésticas asociado a un bajo nivel educativo persistiendo una inequidad de género, relación de poder entre la mujer y la pareja (varón) con un vínculo familiar, donde el varón es el que provee y la mujer la cuidadora de la familia (7). Sin embargo, otros estudios indican que la violencia por parte de la pareja es independiente de la participación económica de la mujer en el hogar; aunque muchas ganan independencia económica, tienen el arraigo de lo enseñado por la familia acerca de la dominación del hombre sobre la mujer, por lo que, en muchas ocasiones, se dejan someter (17).

La necesidad de seguridad y protección se presentó en el $100 \%$ de las participantes, la seguridad se ve afectada por la vulnerabilidad de la mujer frente a factores que ponen en riesgo su vida, la intención de las mujeres al 
denunciar la violencia vivida es focalizada en su necesidad de protección y justicia frente a la inseguridad vivida (18). Se infiere que la necesidad de seguridad está relacionada a la protección que solicitan las mujeres, a que el agresor sea llevado preso por sus actos y maltrato, a pesar que muchas veces desisten de la denuncia; pero exigen el respeto por la ley y el cumplimiento de la misma pues la mujer se ve desprotegida en su seguridad en el hogar, al daño físico o lesiones producto de la violencia, con la necesidad de proteger a los hijos y muchas veces se sienten amenazadas por el hombre en su condición de agresor.

Los resultados pueden ser explicados porque se considera que la mujer tiene necesidad de seguridad y protección pues el riesgo de afrontar día a día situaciones de control o dominación en un entorno de violencia por parte de la pareja puede desencadenar situaciones cada vez más violentas, con actos agresivos sin que prevalezca la autonomía, con actos que son considerados por la Ley de protección contra la violencia familiar como una vulneración a los derechos humanos de las mujeres, los resultados sugieren una intervención de todas las instancias a pesar que la opinión generalizada sigue siendo que los problemas familiares se resuelven en el ámbito privado.

Respecto a la necesidad de comunicarse que se presenta en el $100 \%$ de mujeres afectadas, hallazgo que involucra expresar emociones, necesidades, temores $u$ opiniones en relación a la violencia vivenciada, y que no ha sido abordado por otros estudios, sin embargo, en un estudio cualitativo se señala, que la violencia hacia la mujer muestra consecuencias en las víctimas, como el miedo, el terror, desconfianza y poseen una personalidad introvertida, reservadas, calladas, o con tendencia a ser menos sociables con expresión facial de ansiedad, angustia y miedo a comunicar la violencia vivida con tendencia al distraimiento, y estado de hipervigilancia (15). Existe la necesidad de comunicar la situación de violencia vivida a quienes consideran pueden ayudarlas siendo los familiares como la madre, hermanos, amigos o vecinos, sin embargo algunas mujeres prefieren mantener los hechos en el ámbito privado. Siendo necesaria la intervención de una tercera persona para frenar la violencia perpetrada.

A pesar de contar con instancias como la comisaria, o establecimiento de salud donde acuden las mujeres víctimas, sin embargo sus demandas no son atendidas según sus necesidades. La mayoría de las mujeres que padece algún tipo de violencia acuden asiduamente a los servicios sanitarios con demandas de distinto tipo, por ello, los/as profesionales de la salud, son un valioso recurso para la detección y el abordaje de la violencia familiar (16). Al respecto muchas mujeres no aceptan pasivamente la violencia y el contar su experiencia a otras personas y la búsqueda activa de servicios son algunas formas de enfrentar la violencia, comunicando los hechos.

Respecto a la necesidad de cuidado de vivir según sus creencias/valores en el estudio de Meléndez se halló que más de la mitad de las entrevistadas reconocieron el derecho a la igualdad como el más importante; y en relación a los mitos y creencias sobre el inicio de la violencia se aúna la permanencia de valores tradicionales sobre la mujer, como la cuidadora de la familia, la protectora, la justificadora, la paciente y tolerante (17). En ese sentido la crianza recibida así como las creencias forman parte del entorno cultural en donde se desenvuelve la mujer, que condiciona sujeciones e ideas que la predisponen a sufrir violencia, la valoración cultural constituye un factor que está implícito en las decisiones que toma la mujer respecto a la aceptación de la violencia, estos valores tradicionales justifican la violencia vivida en un entorno que la refuerza cotidianamente. Respecto a la necesidad trabajar y realizarse el 90,5\% muestra esta necesidad. Durante la violencia vivida las mujeres sienten la frustración de no poder realizarse por las condiciones de poder, insatisfacción consigo mismas frente a la autorrealización. De tal manera que las mujeres están desprovistas de oportunidades y con inequidades en sus roles de género.

La necesidad de participar en actividades recreativas reportó un $62.2 \%$ y la necesidad de aprendizaje un $56.8 \%$. Según Virginia Henderson la necesidad de ocio y recreo, constituye un requisito fundamental indispensable para mantener la integridad. Asimismo para la necesidad de aprendizaje que responden a la necesidad de estudiar, descubrir o satisfacer la curiosidad que conduce a un desarrollo normal de la salud, la adquisición de conocimientos y adiestramiento (11). Sin embargo en las mujeres afectadas por violencia estas necesidades no son valoradas como prioritarias y esta situación puede empeorar o mejorar según el nivel educativo que posea la mujer (18).

Cabe mencionar que en el caso de la mujer afectada por violencia el aislamiento y la situación de violencia vivida limita su actuación por lo tanto no se generan espacios de recreación en su entorno social así como la limitación para el aprendizaje influenciada por limitaciones de su estado emocional, la motivación interna o externa, la edad, el nivel de instrucción, situación importante de ser abordada en atención a las necesidades de las víctimas.

En cuanto ha la variable funcionamiento familiar según APGAR se evidenció que más de la mitad de mujeres presentó disfunción familiar severa, en ese sentido las 
familias que presentaron algún nivel de disfuncionalidad representa el 98,6\%. Otros datos similares muestran que en la mayoría de los casos de violencia intrafamiliar se presenta algún nivel de disfuncionalidad, lo cual indica que los desajustes adaptativos en el entorno familiar influyen al momento de tomar decisiones para denunciar la violencia vivida (6).

En estudios sobre disfuncionalidad familiar se asocia a una estructura familiar multigeneracional disfuncional con violencia familiar, asimismo, en el hogar disfuncional se suelen encontrar problemas de índole psicosocial como alcoholismo, conducta antisocial, promiscuidad, conducta suicida y métodos incorrectos de crianza, en un escenario en el que las mujeres han sido históricamente víctimas sin un proceso de intervención desde la familia que sigue patrones de crianza a lo largo de su historia con una elevada tendencia a reproducir elementos de los que también fueron víctimas, donde la violencia se perpetua a traves de las generaciones, el ciclo de violencia y este patrón de conducta en un contexto familiar toma a un padre violento como modelo de rol. Por esa razón en las relaciones disfuncionales se explicaría que las mujeres aprenden que la violencia es algo habitual porque se ha perpetrado a lo largo de generaciones en sus familias $(6,19)$.

Al relacionar las variables de estudio se evidenció la relación indirecta y significativa entre las necesidades de cuidado y el funcionamiento familiar en mujeres afectadas por violencia (Grafico 1) donde el coeficiente de correlación Rho de Spearman Rho= -0.240; muestra una correlación negativa entre ambas variables, la cual resultó estadísticamente significativa $\mathrm{p}=0.039$; es decir, a niveles altos de necesidades de cuidado se corresponden con niveles bajos de funcionamiento familiar y niveles bajos de necesidades de cuidado corresponde con niveles altos de funcionamiento familiar. Este hallazgo muestra una asociación importante, sin embargo en diversos estudios las variables han sido abordadas de manera independiente y correlacionadas con otras variables similares, se destaca que la disfuncionalidad familiar es el correlato del mal funcionamiento de otros sistemas sociales y de acuerdo a los hallazgos la necesidad de adaptación de las mujeres se ha visto afectada por la violencia intrafamiliar y la relación entre variables mostró una correlación entre adaptación y necesidad de participación familiar con $99 \%$ en situaciones de tensión, lo que genera al interior del sistema un estado de crisis constante frente a las necesidades básicas (20).

Se deduce que las necesidades de cuidado en familias con disfuncionalidad es permanente pero poco visible con necesidades no cubiertas según sea la severidad de la disfuncionalidad, por lo tanto el abordaje de la intervención con la familia desde el funcionamiento familiar considerando que a menor funcionalidad familiar mayor será las necesidades de cuidado desde la seguridad, protección, verbalización del problema, valores, creencias en torno a la violencia, oportunidad de realizarse como familia, se relaciona con la poca visualización del problema como una prioridad para el desarrollo de la familia.

Finalmente, la necesidad de cuidado y el funcionamiento familiar representan dos variables importantes de seguir siendo estudiadas si deseamos realmente una nueva concepción dirigida hacia la gestión del cuidado para mejorar nuestras intervenciones en mujeres afectadas por violencia, que día a día viven esta situación en su entorno familiar disfuncional. El profesional de Enfermería tiene un reto importante en el marco de la gestión del cuidado el cual es viabilizar la valoración inicial de las mujeres afectadas por violencia intrafamiliar, individualizando a la persona con una atención humanista e integral desde el modelo de Virginia Henderson, con las necesidades de cuidado que requieren intervenciones plasmadas en el proceso de atención de enfermería, asimismo, articular redes de apoyo y gestionando la intervención oportuna desde el primer nivel de atención.

\section{REFERENCIAS BIBLIOGRÁFICAS}

1. Organización Mundial de la Salud. Informe estudio multipaís de la OMS sobre: salud de la mujer y violencia doméstica contra la mujer. informe de un grupo científico de la OMS. Ginebra: OMS; 2005. Serie de Informe Técnico: 309.

2. Ministerio de la mujer y poblaciones vulnerables. Documento técnico Lineamientos éticos para las investigaciones en violencia familiar y sexual Lima: MIMP. [Internet]. 2014 [citado el 15 octubre 2014]. Disponible en http://www.mimp.gob.pe/files/ transparencia/resoluciones_ministeriales/anexo_ rm_338_2014-lineamientos.pdf

3. Instituto Nacional de Estadística e informática y movimiento manuela ramos. Documento técnico: Brechas de género: insumos para la adopción de medidas a favor de las mujeres. 2010. numero de publicación 02831 .

4. Encuesta Demográfica y de Salud Familiar- ENDES 2013. Instituto Nacional de Estadística e Informática. Lima-Perú, [Internet]. 2014 [Citado el 10 agosto 2014]. Disponible en http://www.inei.gob.pe/media/ 
MenuRecursivo/publicaciones_digitales/Est/Lib1151/ index.html

5. Organización Mundial de la Salud. Estimaciones mundiales y regionales de la violencia contra la mujer. Prevalencia y efectos de la violencia conyugal y de la violencia sexual no conyugal en la salud. Número de referencia OMS: 9789241564625. Ginebra: OMS [Internet]. 2013 [citado el 10 de Julio del 2014]. Disponible en: http://apps.who.int/iris/ bitstream/10665/85243/1/who_rhr_hrp_13.06_spa. pdf?ua $=1$

6. Ministerio de la Mujer y Poblaciones Vulnerables. Programa Nacional contra la violencia familiar y sexual. Perú: Unidad de Generación de Información y Gestión del Conocimiento. Boletín de resúmenes estadísticos [Internet]. 2014. [citado el 16 agosto de 2014]. Disponible en: http://www.mimp.gob. pe/files/programas_nacionales/pncvfs/estadistica/ boletin_julio_2014/boletin_julio_2014.pdf

7. Gonzales J, Rivas F, Marin X, Villamil L. Niveles de disfunción familiar, en veinte mujeres víctimas de violencia intrafamiliar en el municipio de ArmeniaColombia. Ágora usb [Internet]. 2013 [citado el 21 agosto]; vol.13(2): 399-410. Disponible en: http://www.scielo.org.co/scielo.php?script=sci arttext\&pid $=$ s1657-80312013000200005\&lng=en \& nrm $=$ iso

8. Henderson V. Desarrollo de un concepto personal. En: Naturaleza de la enfermería, reflexiones 25 años después. Editorial Interamericana. McGraw-Hill; México, DF. 1994.

9. García A, Francisco del Rey C, Palazuelos P, De Bustos M. El modelo conceptual de Virginia Henderson y la etapa de valoración del proceso de atención de enfermería. Rev. Educare 21. [Internet]. 2005. [citado el 18 agosto 2014]; Disponible en: http://www.enfermeria21.com/revistas/educare/ articulo/9024/

10. Ferraz M., Lacerda M., Labronici L., Maftum M, Raimondo M. O cuidado de enfermagem a vítimas de violência doméstica. Cogitare enferm. [Internet]. 2010. [citado 2014 ago 18]; 14(4): 755-759. Disponible en: http://www.revenf.bvs.br/scielo. php?script $=$ sci_arttext\&pid $=$ s141485362009000400 022\&lng=es

11. Rúa A, Pita R, Gómez I. Satisfacción de las necesidades básicas en la población inmigrante femenina marroquí en Arteixo- España.. Rev. Esc. Enferm. Usp. [Internet]. 2010 junio [citado 21 de agosto 2014]; 44 (2):249-256. Disponible en: http://www.scielo.br/scielo.php?script=sci_ arttext\&pid $=\mathrm{s} 0080-62342010000200002 \& \operatorname{lng}=\mathrm{en}$. http://dx.doi.org/10.1590/s0080623420100002

12. Bellido J, Lendínez J. Proceso enfermero desde el modelo de cuidados de Virginia Henderson y los lenguajes N. Colegio Oficial de Enfermería de Jaén -España [internet] 2010. [Citado el 09 de marzo 2012]. Disponible en: http://www.enfermeriajaen.com/index. php/publicaciones/cuadernos-de-enfermeros

13. Alegre Y, Suárez M. Instrumentos de atención a la familia: El familiograma y el Apgar familiar. Rampa [Internet]. 2006. [citado el 29 agosto 2012]; 1(1):4857. Disponible en http://www.idefiperu.org/ramnro1/ n9cpg48cadec\%20instrum\%20familia2a.pdf

14. Suarez M, Alcalá M. APGAR familiar: una herramienta para detectar disfunción familiar. Rev Med La Paz [internet]. 2014 enero-junio [citado el 21 de diciembre 2014]; 20(1); Disponible en: http://www.scielo.org. bo/scielo.php?pid=S172689582014000100010\&scr ipt=sci_arttext

15. Bardales O. Estado de las investigaciones en violencia familiar y sexual 2006 - 2010. Programa Nacional contra la Violencia Familiar y Sexual. MIMP, LimaPerú: Sagitario Editores; 2012

16. Arredondo A, Broco M, Alcalá Teresa, Rivera A, Jiménez I, Gallardo C. Profesionales de atención primaria de Madrid y violencia de pareja hacia la mujer en el año 2012. Rev. Esp. Salud pública, Madrid. [Internet] 2012.[citado el 10 agosto 2014]; 86 (1): 85-89. Disponible en: http://scielo.isciii.es/scielo. php?script $=$ sci_arttext\&pid $=$ s 113557272012000100 008\&lng=es

17. Meléndez L, Rosas C, Yáñez G, Entre luces y sombras, caminos para acceder a la justicia. Estudio de la comisaría de mujeres de Villa el Salvador. Lima: Centro de la Mujer Peruana Flora Tristán y Movimiento Manuela Ramos. [Internet]. 2010 [citado el 13 de noviembre 2012]. Disponible en: http:// www.manuela.org.pe/wp-content/uploads/2010/06/ Entre_Luces_y_Sombras.pdf

18. Cabrera M, Poll H, Mederos M. Violencia contra la mujer en la comunidad. Medisan [Internet]. 2012 [citado 10 julio 2014] ; 16(8): 1267-1273. Disponible 
en: http://scielo.sld.cu/scielo.php? script $=$ sci arttext\&pid $=$ s1029-30192012000800012\&lng=es

19. Bellido J., Lendínez J. Proceso enfermero desde el modelo de cuidados de Virginia Henderson y los lenguajes N. Colegio Oficial de Enfermería de Jaén -España [Internet]. 2010. [Citado el 09 de marzo 2012]. Disponible en: http://www.enfermeriajaen.com/index. php/publicaciones/cuadernos-de-enfermeros.
20. Organización Mundial de la Salud y Escuela de Higiene y Medicina Tropical de Londres. Prevención de la violencia sexual y violencia infligida por la pareja contra las mujeres: qué hacer y cómo obtener evidencias. [En internet]. Washington, DC: Organización Panamericana de la Salud, 2011.[Citado el 12 de julio 2014].Disponible en: http://www.who. int/violence_injury_prevention/violence/activities/ intimate/en/

Fecha de recibido: 15 de Octubre de2014

Fecha de aceptación: 29 de Noviembre de 2014. 